\title{
A Dual-Band Band-Pass Filter with Overlap Step-Impedance and Capacitively Loaded Hairpin Resonators for Wireless LAN Systems
}

\author{
P. Chomtong, C. Mahatthanajatuphat, and P. Akkaraekthalin \\ Department of Electrical Engineering, Faculty of Engineering, King Mongkut's University of Technology North Bangkok, \\ 1518 Pibulsongkram Road, Bangsue, Bangkok 10800, Thailand \\ Correspondence should be addressed to P. Chomtong, tonkmutnb@hotmail.com
}

Received 10 November 2010; Revised 1 February 2011; Accepted 1 March 2011

Academic Editor: Yo Shen Lin

Copyright () 2011 P. Chomtong et al. This is an open access article distributed under the Creative Commons Attribution License, which permits unrestricted use, distribution, and reproduction in any medium, provided the original work is properly cited.

This paper presents a dual-band band-pass filter using modified cross-coupled step-impedance and capacitively loaded hairpin resonators for WLAN systems. The proposed filter has been designed to operate at a fundamental frequency of $2.4 \mathrm{GHz}$ and the first harmonics frequency of $5.2 \mathrm{GHz}$. The techniques of step impedance and load capacitor are combined in the design of the proposed filter. In particular, the techniques of modified cross-coupling and overlap resonators are applied to improve the response of insertion losses $S_{21}$ at the first harmonic frequency of $5.2 \mathrm{GHz}$. The simulated and experimental results of insertion losses and return losses are better than $3 \mathrm{~dB}$ and $20 \mathrm{~dB}$, respectively, at the operating frequencies.

\section{Introduction}

Wireless communication systems have been growing rapidly in many applications. Wireless Local Area Networks (WLAN) are widely used in many countries. The most widely used standard WLAN on IEEE $802.11 \mathrm{~b} / \mathrm{g} / \mathrm{a}$ is provided at the operating frequencies of $2.4 \mathrm{GHz}, 5.2 \mathrm{GHz}$, and $5.8 \mathrm{GHz}$, respectively. A band-pass filter is an important device which is composed in the front end of WLAN systems. Bandpass filters have been researched and developed with many materials. There has been keen interest in microstrip bandpass filters also, due to ease design, compact size, and low cost.

Currently, several techniques such as a step-impedance technique [1-8], a capacitive load technique [9-13], and a dual-mode technique [14-19] have been applied to design the resonators for band-pass filters. The step-impedance resonator (SIR) [1-4] can shift the first harmonic of resonant frequency to any frequency, due to the different ratio between the impedances of resonator. Also, a step-impedance resonator can be redesigned as a hairpin line resonator in order to reduce size to the electrical length of approximately $\lambda_{g} / 2$. Additionally, the capacitive load technique [9-12] can be applied to design the resonators for reduced size and shifting of the first harmonic of resonant frequency to any frequency, the effects of which are achieved by changing the capacitive load. However, it is important to design the coupling between resonators of band-pass filters. The most typical coupling between resonators include parallel, hairpin line resonator and cross-couplings [14-16]. In particular, the cross-coupling can improve the characteristics of band-pass filters, such as shape of insertion loss and transmission zero.

This paper presents a dual-band band-pass filter using modified cross-coupled step-impedance and capacitively loaded hairpin resonators for WLAN system, which is designed to operate at the fundamental and first harmonic of resonant frequencies of $2.4 \mathrm{GHz}$ and $5.2 \mathrm{GHz}$, respectively. The techniques of step-impedance, capacitively loaded, and modified cross-coupling are applied to design the proposed filter. Moreover, the overlap resonators technique resulting in the coupling effect of our proposed filter will be investigated by simulation using full-wave method of moment (MOM) software package from IE3D [20] at the resonant frequencies.

The organization of this paper is as follows. In Section 2, a brief explanation on the proposed filter design will be given. Then, the investigation and experiment of the properties for 


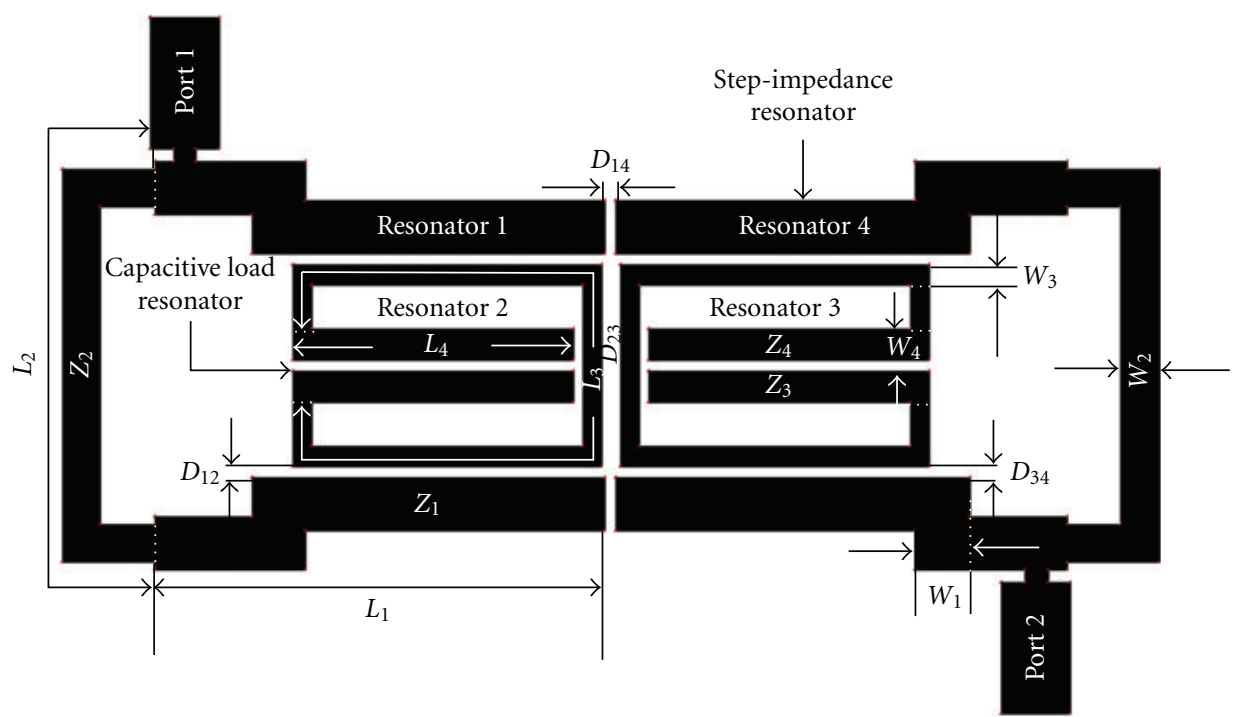

FIgURE 1: The configuration of the proposed filter with modified cross-coupled band-pass filter.

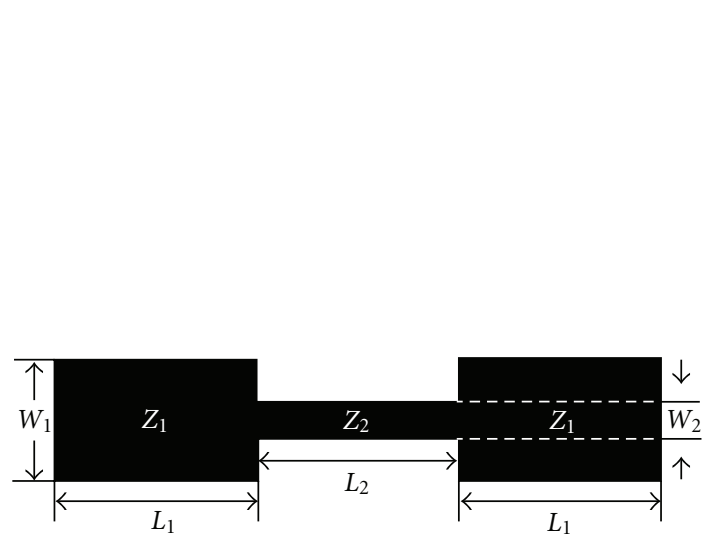

(a)

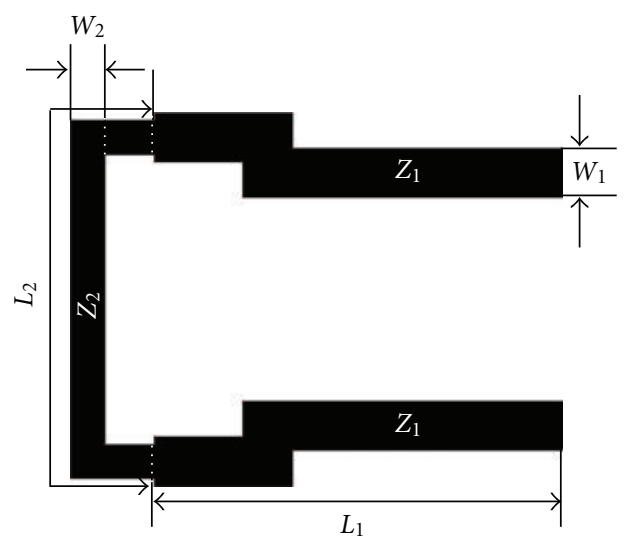

(b)

FIGURE 2: Configuration of step-impedance line resonator. (a) Basic structure of SIR. (b) Folded SIR.

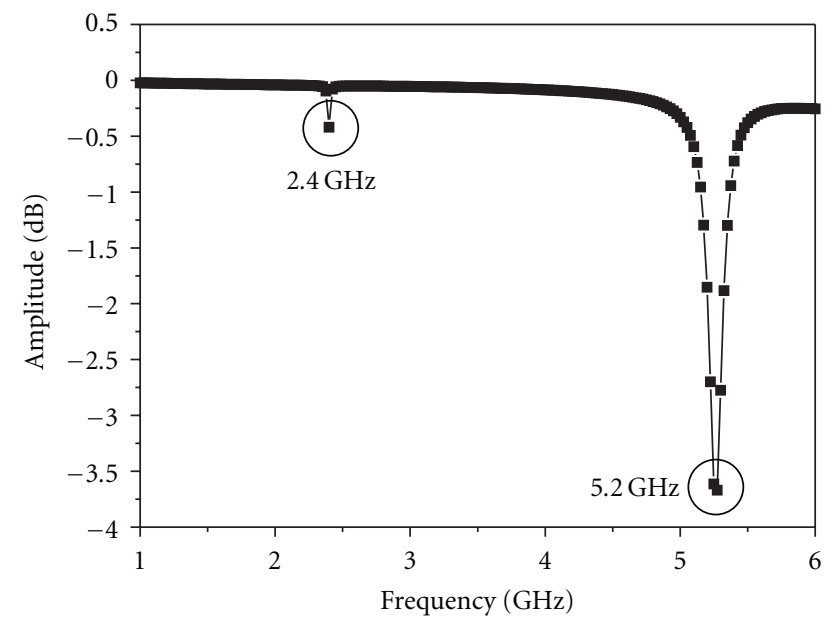

$-S_{1,1}(\mathrm{~dB})$

FIgURE 3: The simulated results of folded SIR. 


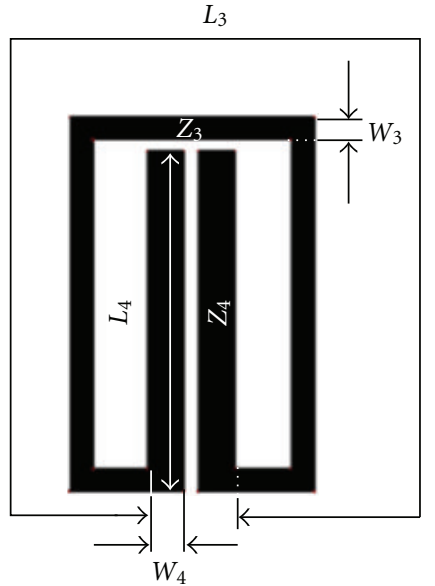

(a)

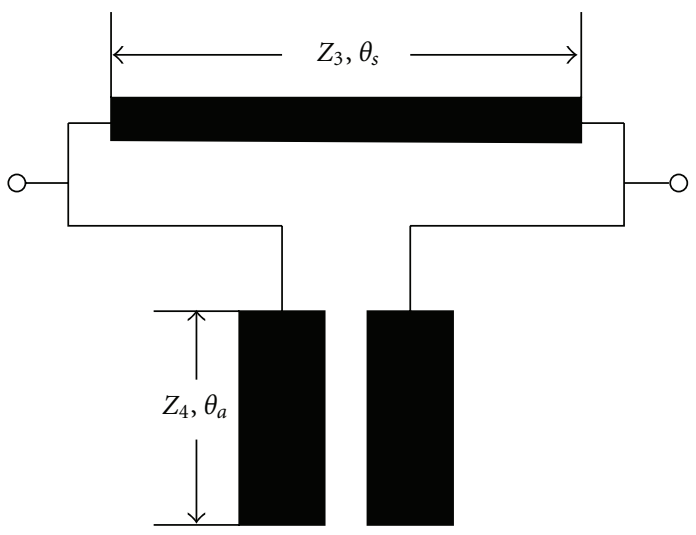

(b)

FIGURE 4: Schemetic of the miniaturize hairpin resonator and equivalent circuit of SIR. (a) Geometrical diagram of the miniaturized hairpin resonator and (b) a simplified equivalent circuit of SIR with internal coupled line.

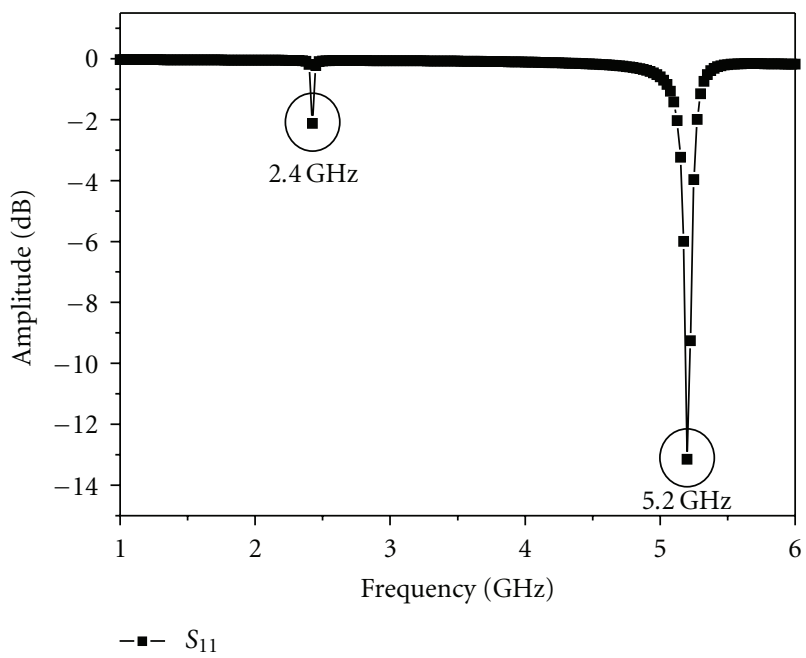

FIgURE 5: The result of simulation $l$ to examine a resonance of capacitively loaded transmission.

coupling between resonators of the filter will be discussed in Section 3. Finally, the results are discussed in Section 4.

\section{Design of the Proposed Filter}

Figure 1 shows the modified cross-coupled band-pass filter, consisting of the step-impedance resonators 1 and 4 and the inner capacitively loaded resonators 2 and 3 , respectively. The proposed filter is fabricated on GML 1000 dielectric substrate with a thickness of $0.762 \mathrm{~mm}$, a relative permittivity of 3.2 $\left(\varepsilon_{r}\right)$, and a loss tangent of 0.004 . The design of the stepimpedance and capacitively loaded resonators are illustrated below.

Figure 2(a) shows the configuration of the SIR basic structure, which is created to a folded SIR as shown in Figure 2(b). The folded SIR is designed to operate at the fundamental and first harmonic resonant frequencies of $2.4 \mathrm{GHz}$ and $5.2 \mathrm{GHz}$, respectively. The folded SIR was defined by the impedance ratio between impedance $Z_{2}$ and impedance $Z_{1}[1-3]$. In this paper, the impedances $Z_{2}=70 \Omega$ and $Z_{1}=58 \Omega$ are chosen, resulting in the operating frequency of the first harmonic resonant frequency at $5.2 \mathrm{GHz}$. Also, the appropriate parameters of the folded SIR, as shown in Figure 2 , are $W_{1}=1.43 \mathrm{~mm}, L_{1}=12.15 \mathrm{~mm}, W_{2}=1 \mathrm{~mm}$, and $L_{2}=11.25 \mathrm{~mm}$. Therefore, the results of return loss $\left(S_{11}\right)$ are computed by simulation software IE3D, as shown in Figure 3. It can be clearly seen that the resonant frequencies are occurred at 2.4 and $5.2 \mathrm{GHz}$, respectively.

The schematic and equivalent circuits of the capacitively loaded resonators are depicted in Figures 4(a) and 4(b), respectively, which are designed on fundamental frequency at $2.4 \mathrm{GHz}$ and first harmonic frequency at $5.2 \mathrm{GHz}$ as the same as step-impedance resonators. Especially, $Z_{3}, \theta_{s}$ are the characteristic impedance and electrical length of transmission line and $Z_{4}, \theta_{a}$ are the characteristic impedance and electrical length of coupled line. Also, the loaded 


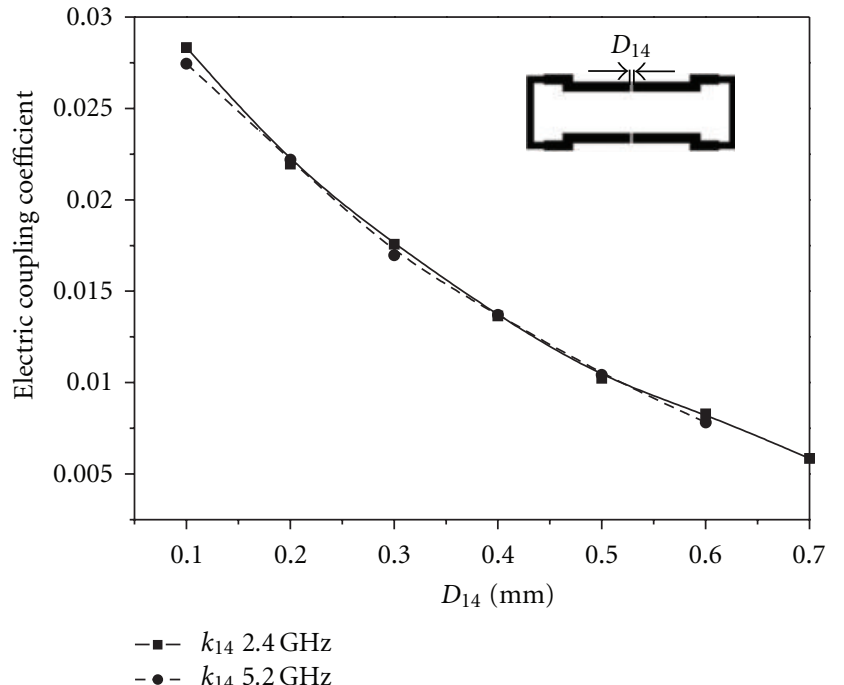

(a)

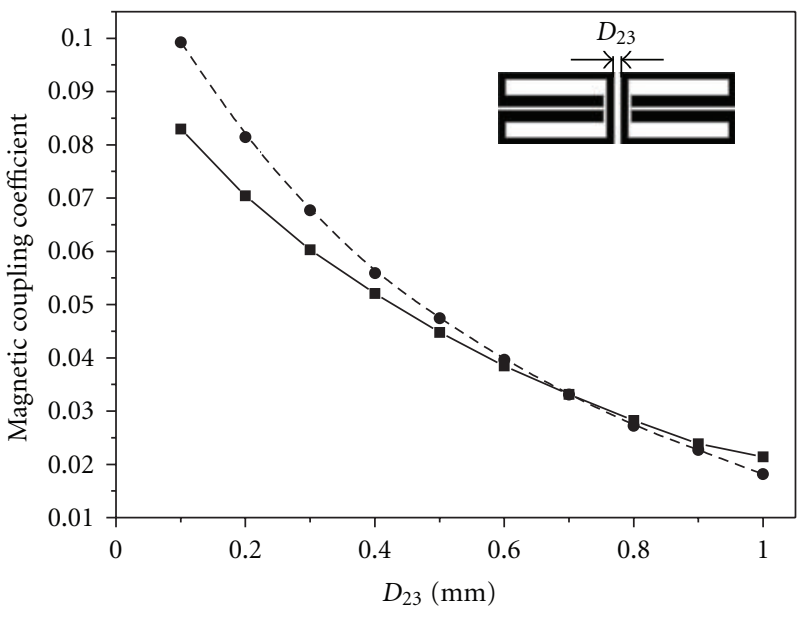

$-k_{23} 2.4 \mathrm{GHz}$

- • $k_{23} 5.2 \mathrm{GHz}$

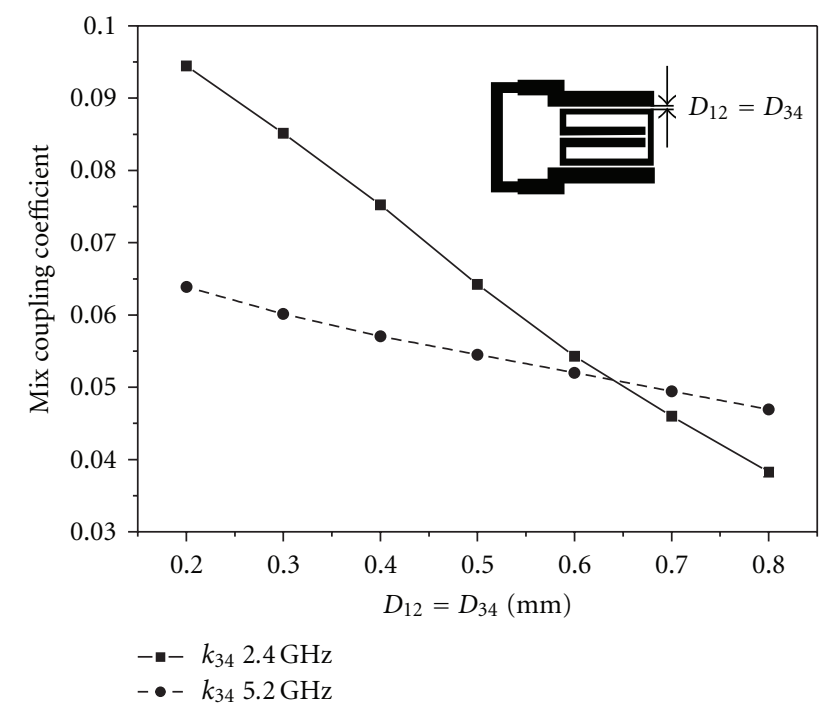

(c)

FIGURE 6: Typical coupling coefficients of the proposed filter without overlap resonators (a) electric coupling coefficients, (b) magnetic coupling coefficients, and (c) mixed coupling coefficients.

capacitance $C_{L}$ which occurs by coupling between the impedance of coupled line is calculated in (1). Following the results in $[10,12]$, the loaded capacitance $C_{L}$ can reduce the electrical length of transmission line from $\lambda / 2$ to $\lambda / 4$ smaller than step-impedance resonators. In addition, it is concordant with first harmonic frequency that made desired frequency of resonators as (2) and (3). The $f_{0}, f_{H 1}, \theta_{S 0}$, and $\theta_{S 1}$ are fundamental frequency, first harmonics frequency, electrical length at the fundamental frequency, and electrical length at first harmonics frequency, respectively. The simulated results of capacitively loaded resonator show that the resonant frequencies are at 2.4 and $5.2 \mathrm{GHz}$, as illustrated in Figure 5.

$$
C_{L}=\frac{\tan \theta_{a}}{Z_{4} \omega}
$$

$$
\begin{gathered}
\theta_{S 0}=2 \tan 1\left(\frac{1}{\pi f_{0} Z_{3} C_{L}}\right), \\
\theta_{S 1}=2 \pi-2 \tan ^{-1}\left(\pi f_{H 1} Z_{3} C_{L}\right) .
\end{gathered}
$$

Therefore, the appropriate parameters of the capacitively loaded resonator are as follows: $W_{3}=0.5 \mathrm{~mm}, W_{4}=$ $1.255 \mathrm{~mm}, L_{3}=7.6 \mathrm{~mm}, L_{4}=7.2 \mathrm{~mm}$, and $L_{5}=6.41 \mathrm{~mm}$ to operate at the fundamental and first harmonic resonant frequencies of $2.4 \mathrm{GHz}$ and $5.2 \mathrm{GHz}$, respectively.

Finally, the modified cross-coupling technique $[15,16]$ is used in the proposed filter as shown in Figure 1. The four resonators are properly placed in the structure for appropriate coupling to obtain a pattern of a Chebyshev ripple constant 0.001 . The fractional bandwidth of the fundamental frequency band of $83 \mathrm{MHz}(3.45 \%)$ and the 


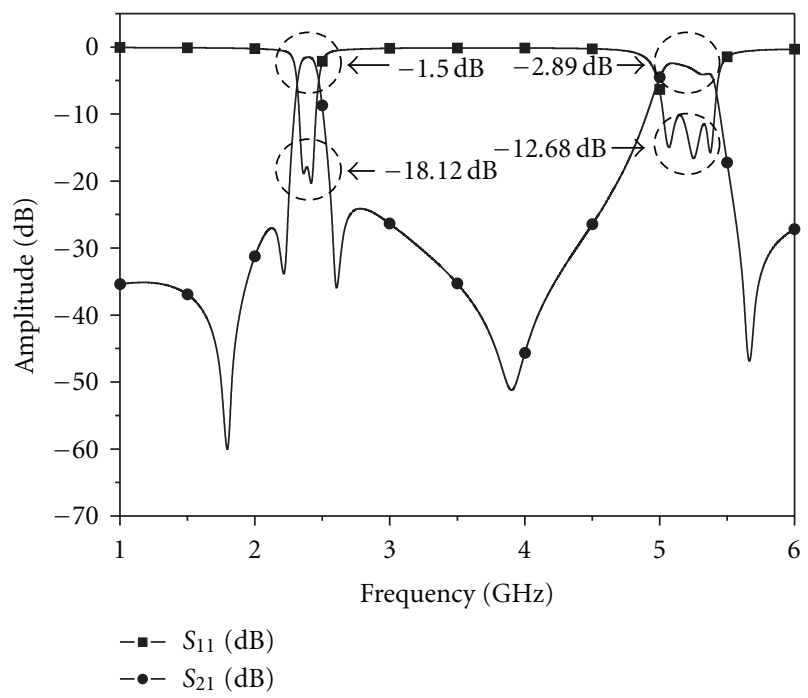

FIGURE 7: Simulated responses of the proposed filter without overlap resonator.

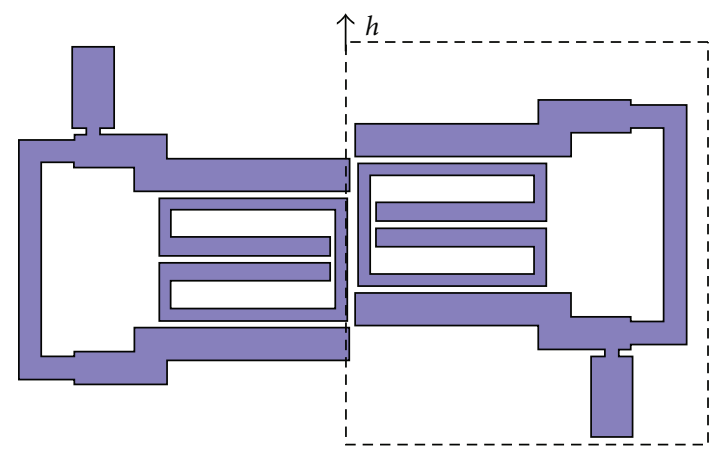

(a)

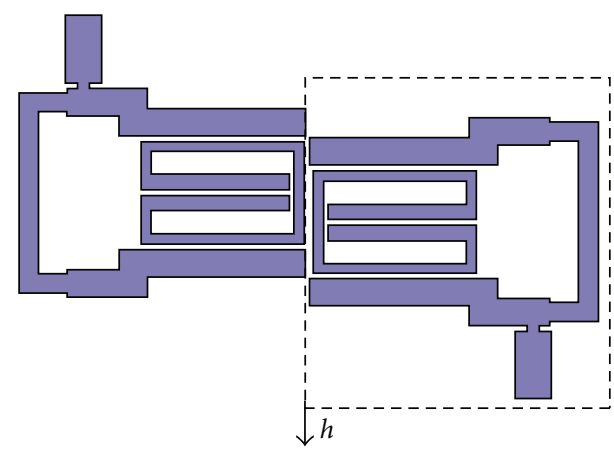

(b)

FIGURE 8: The proposed filter with overlap distance $h$ between resonators along $y$ axis (a) shifted up and (b) shifted down.

fractional bandwidth of the $5.2 \mathrm{GHz}$ band of $200 \mathrm{MHz}$ $(3.84 \%)$ are used to calculate the coupling coefficient $k_{i j}$. The low-pass prototype of the proposed filter is calculated and obtained: $g_{0}=1, g_{1}=0.7533, g_{2}=1.16552, J_{1}=$ -0.4513 , and $J_{2}=1.05789$. The external quality factors are $Q_{e i}=Q_{e o}=21.5225$. The coupling coefficients between the adjacent resonators can be found to be $k_{12}=k_{34}=0.037353$, $k_{23}=0.03176$, and $k_{14}=0.020974$, which the coupling coefficients of $k_{12}, k_{34}, k_{23}$, and $k_{14}$ at the resonant frequencies of $2.4 \mathrm{GHz}$ and $5.2 \mathrm{GHz}$ are identical.

The simulated resonant frequency response of the modified cross-coupled resonator structures is illustrated in [16]. The obtained lower and higher resonant frequencies, that is, $f_{a}$ and $f_{b}$, respectively, from the connection between resonators are readily identified by the two split resonant peaks. In order to simulate the frequency responses of these conventional coupling coefficients, the full-wave method of moment (MOM) software package from IE3D was exploited. The coupling coefficient can be graphically approximated from these two resonant frequencies and obtained by using the following equation:

$$
K_{i j}=\frac{f_{b}^{2}-f_{a}^{2}}{f_{b}^{2}+f_{a}^{2}}
$$

The conceivable coupling coefficient curves against the distance $d$ for the electric, magnetic, and mixed couplings, respectively, are depicted in Figure 6. The results show that it can be clearly seen that the coupling coefficients of electric, magnetic, and mixed couplings decrease as the distance $D$ increased. In order to determine the distance $D$, the coupling coefficients $K_{i j}$ are selected at the sufficiently intersection point between the coupling coefficients $K_{i j}$ of $2.4 \mathrm{GHz}$ and $5.2 \mathrm{GHz}$.

When calculated as previously mentioned, the appropriated electric coupling coefficient $k_{14}=0.020974$ is used to determine the distance $D_{14}$ from the Figure 6(a), where the suitable distance $D_{14}$ is approximately $0.23 \mathrm{~mm}$. As depicted 


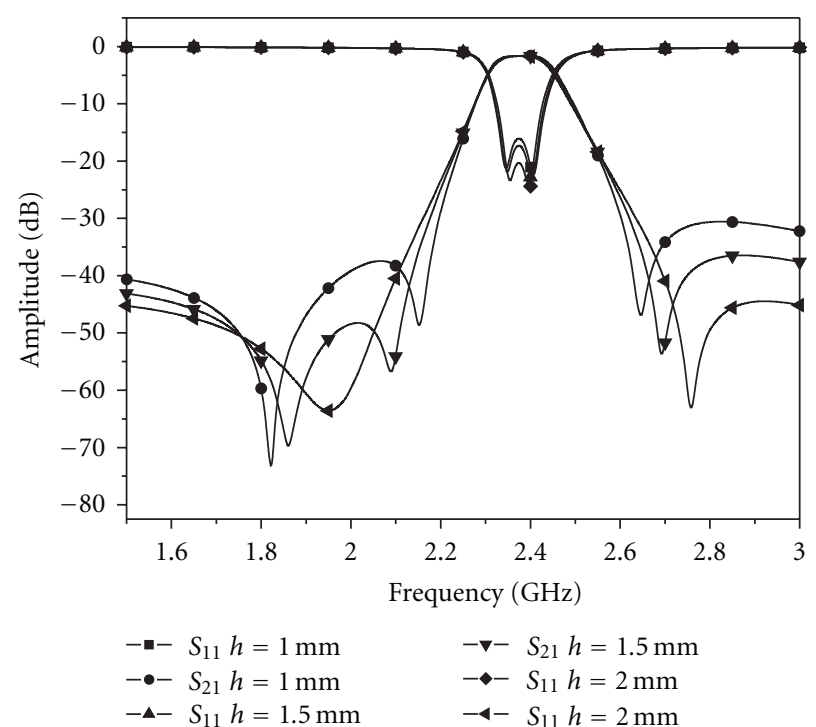

(a)

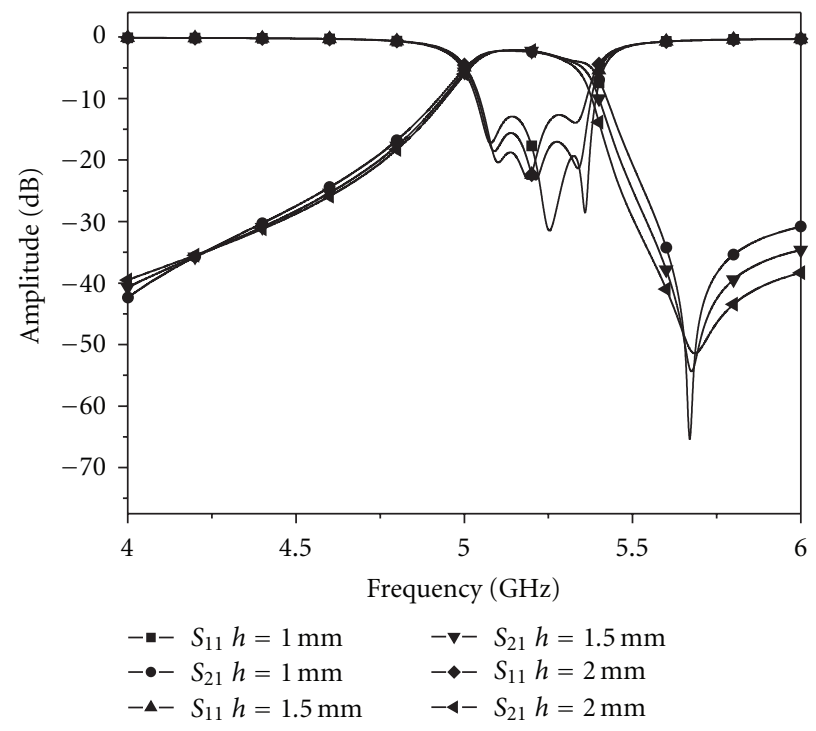

(c)

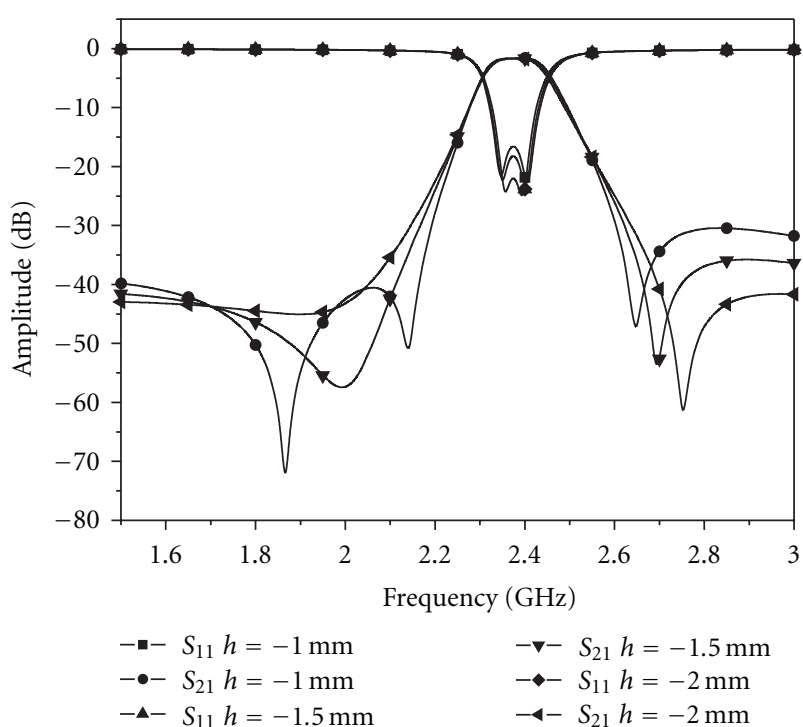

(b)

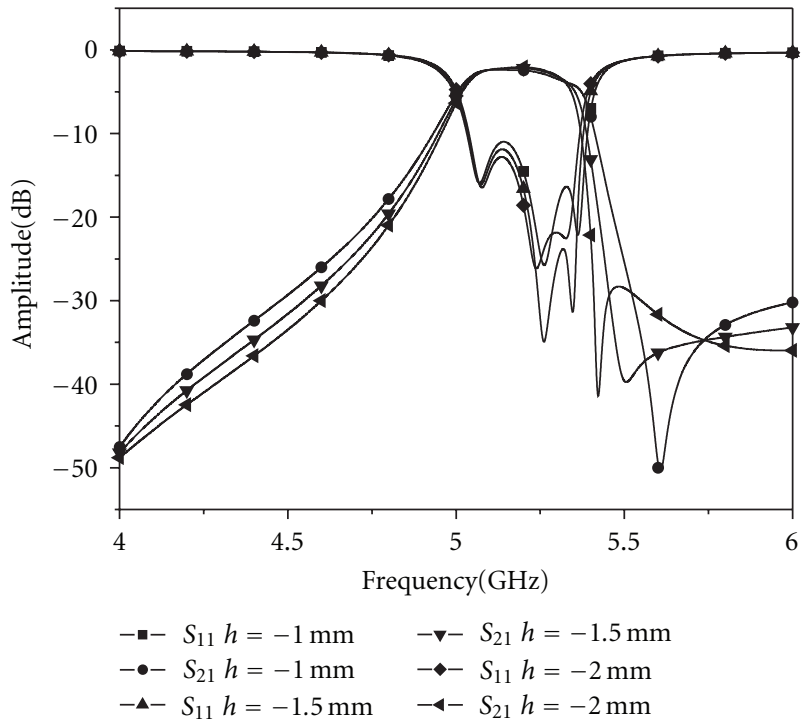

(d)

FIGURE 9: Simulated responses of the proposed filter using overlap resonators technique by varying distance $h$ along (a) $y$ axis at fundamental resonant frequency $2.4 \mathrm{GHz}$, (b) $y$ axis at fundamental resonant frequency $2.4 \mathrm{GHz}$, (c) $y$ axis at fundamental resonant frequency $5.2 \mathrm{GHz}$, and (d) $y$ axis at first harmonics resonant frequency $5.2 \mathrm{GHz}$.

in Figure 6(b), the appropriated magnetic coupling coefficient $k_{23}=0.03176$ is chosen to determine the distance $D_{23}$, which is approximately $0.74 \mathrm{~mm}$. Also, The mixed coupling coefficient $k_{12}=k_{34}=0.037353$ is used to determine the distance $D_{12}$ and $D_{34}$, which are approximately $0.435 \mathrm{~mm}$, as illustrated in Figure 6(c). Therefore, the appropriated parameters $D_{14}=0.23 \mathrm{~mm}, D_{23}=0.74 \mathrm{~mm}$, and $D_{12}=$ $D_{34}=0.435 \mathrm{~mm}$ are used to design the proposed filter for investigation and experiment in the next section.

\section{Implementation and Results}

This section presents and discusses the experiment on the properties of coupling between resonators. In the previous section, the appropriated parameters $D_{14}=0.23 \mathrm{~mm}, D_{23}=$ $0.74 \mathrm{~mm}$, and $D_{12}=D_{34}=0.435 \mathrm{~mm}$ were used to design the proposed filter, which responds to the operating frequency of the fundamental and first harmonic resonant frequencies at $2.4 \mathrm{GHz}$ and $5.2 \mathrm{GHz}$, as depicted in Figure 7 . In this figure, it is obvious that the insertion losses of the fundamental and first harmonic resonant frequencies are $1.51 \mathrm{~dB}$ and $2.89 \mathrm{~dB}$, respectively. Also, the return losses and bandwidth of the fundamental and first harmonic resonant frequencies are $18.12 \mathrm{~dB}, 12.68 \mathrm{~dB}, 100 \mathrm{MHz}$, and $270 \mathrm{MHz}$, respectively. Following the results show in Figure 7, the insertion losses of the first harmonic resonant frequency are nearly $3 \mathrm{~dB}$, resulting from the out-of-phase coupling between each resonator. In order to improve the coupling 

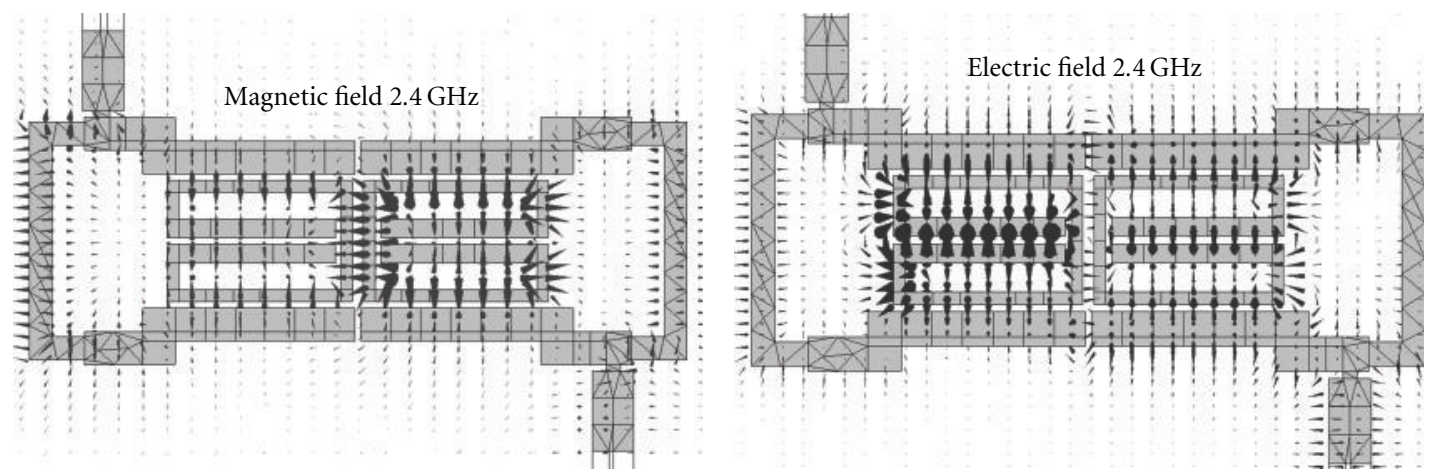

(a)
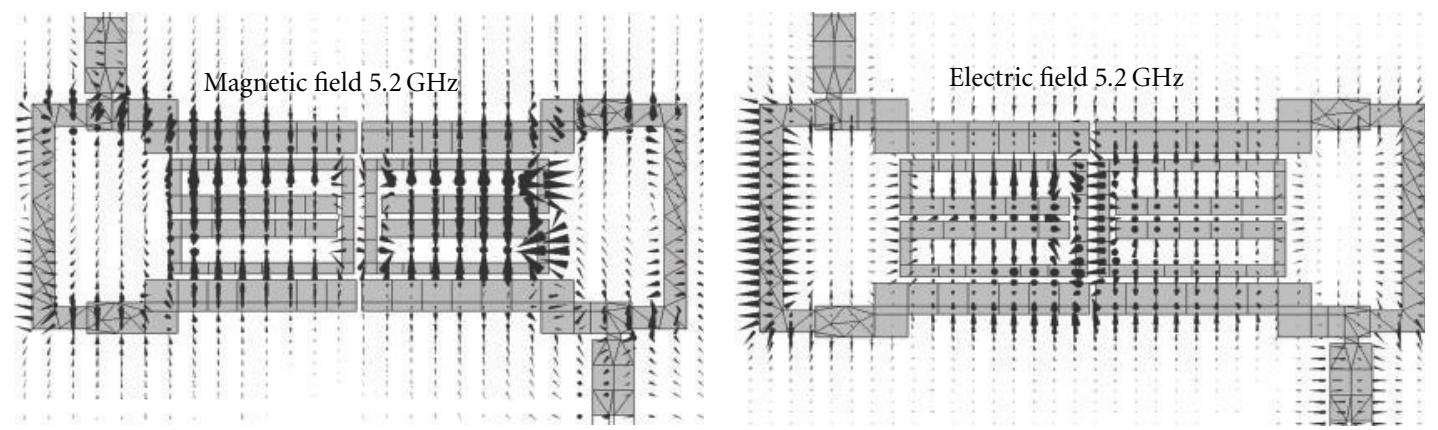

(b)
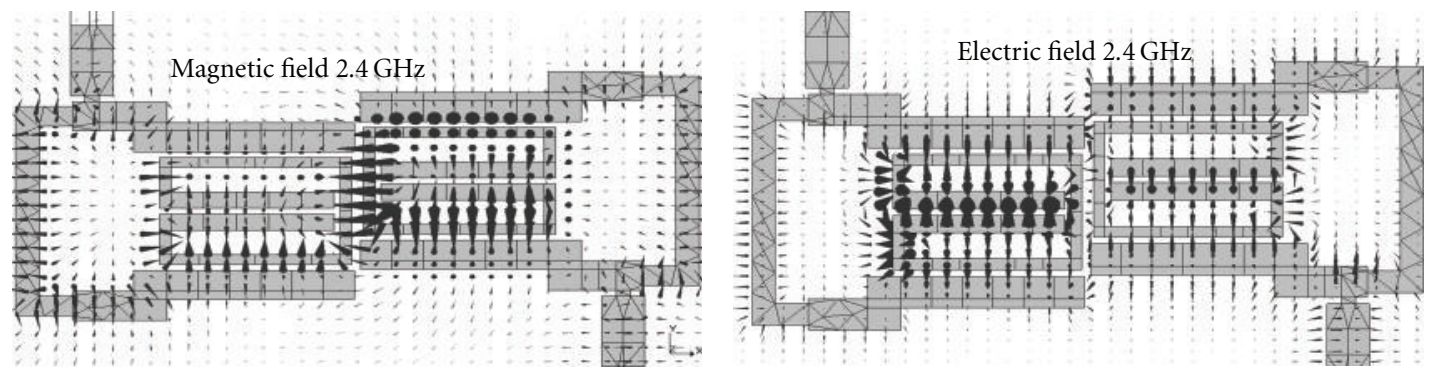

(c)
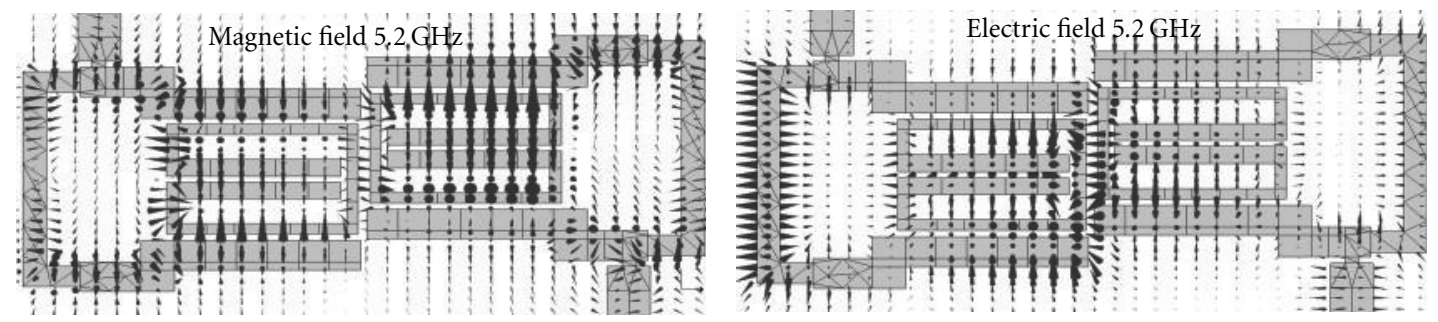

(d)

FIGURE 10: Simulation results of the magnetic and electric fields on the proposed filter at (a) the fundamental resonant frequency of $2.4 \mathrm{GHz}$ without overlap resonator, (b) the first harmonics resonant frequency of $5.2 \mathrm{GHz}$ without overlap resonator, (c) the fundamental resonant frequency of $2.4 \mathrm{GHz}$ with overlap resonator at the distance $h=1.5 \mathrm{~mm}$ along $y$ axis, and (d) the first harmonics resonant frequency of $5.2 \mathrm{GHz}$ with overlap resonator at the distance $h=1.5 \mathrm{~mm}$ along $y$ axis.

between resonators, the overlap resonators method will be used and further investigated.

From the overlap resonators, resonators 3 and 4 will be shifted up and down along the $y$ axis, as illustrated in Figure 8, which the overlap distance of the resonators varied by the parameter $h$, and the parameters of $D_{12}, D_{23}, D_{34}$, and
$D_{14}$ still not changed, held constant. The simulated results of the proposed filter with overlap resonators along $y$ axis are illustrated in Figure 9.

Figures 9(a) and 9(b) show that the return losses and insertion losses at the fundamental resonant frequency are slightly changed by varying the parameter $h$. Also, the return 


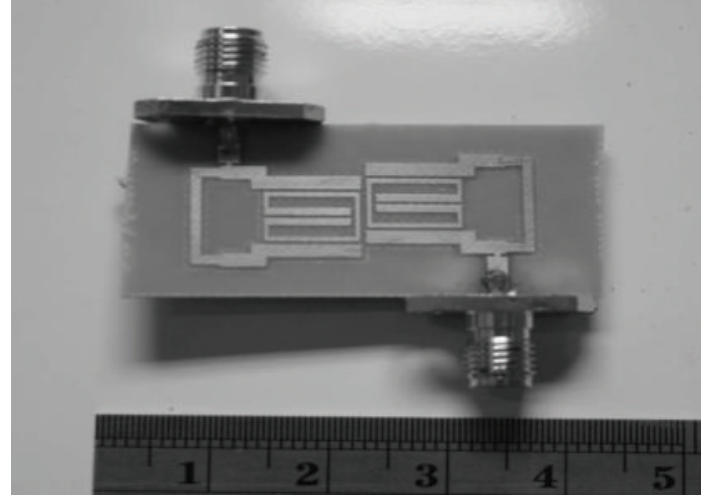

FIgURE 11: Photograph of the proposed filter with overlap resonator.

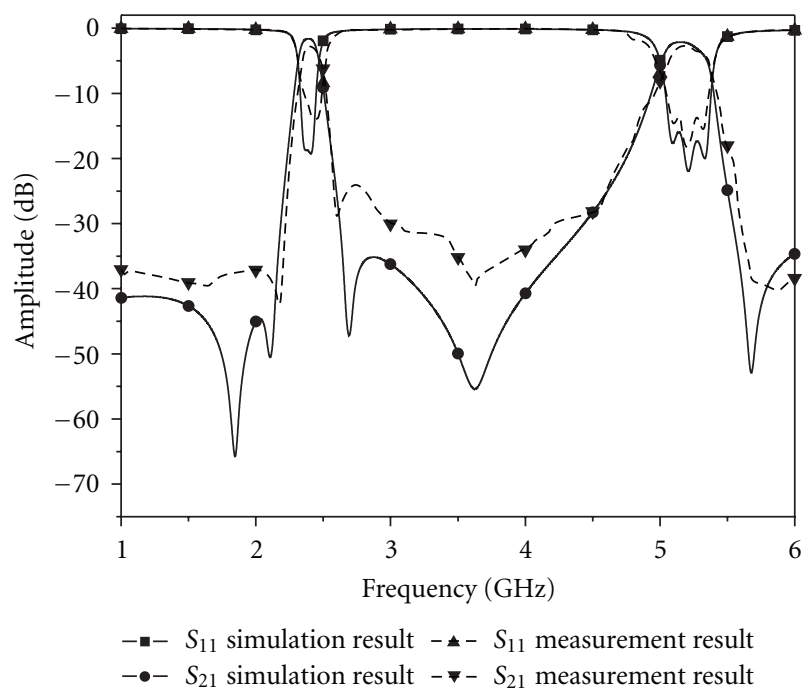

FIGURE 12: Simulated and measured responses of the proposed filter with overlap resonators.

losses and insertion losses at the first harmonic resonant frequency are affected by varying the parameter $h$, as shown in Figures 9(c) and 9(d).

According to the results illustrated in Figures 9(c) and 9(d), it can be clearly seen that the levels of insertion losses in both figures are similar. However, the level of the return losses in Figure 9(c), which are lower than $20 \mathrm{~dB}$, are better than those seen in Figure 9(d), which are higher than $20 \mathrm{~dB}$. Also, the appropriated parameter $h=1.5 \mathrm{~mm}$ is selected to improve the insertion losses at the first harmonic resonant frequency of the proposed filter, as shown in Figure 9(c).

Additionally, the simulated results of electric field on the proposed filter with and without overlap resonators are illustrated in Figure 10.

Figure 10(a) shows the coupling effects of the proposed filter without overlap resonators at the resonant frequency of $2.4 \mathrm{GHz}$. Here, the mix coupling effects are achieved by the coupling between resonators 1 and 2, also, resonators 3 and 4 . The magnetic coupling, as shown in magnetic filed, between resonators 2 and 3 is robust, particularly the electric coupling, as shown in electric field, between resonators 1 and 4 is weak. Therefore, the level of insertion loss at the resonant frequency of $2.4 \mathrm{GHz}$ is lower.

Figure 10(b) shows the coupling effects of the proposed filter without overlap resonators at the resonant frequency of $5.2 \mathrm{GHz}$. It can be clearly observed that the mix and magnetic coupling effects are similar to those illustrated in Figure 10(a) but the magnetic coupling, shown as magnetic field, between resonators 2 and 3 decreases, and also, the level of insertion loss is higher and the electric coupling, shown as electric field, is strong due to the coupling between resonator 2 and 3 at the higher frequency as same as capacitance. Therefore, the coupling between resonator 2 and 3 affects more electrical coupling than magnetic coupling at higher frequency.

The coupling effects of the proposed filter with overlap resonators at the resonant frequency of $2.4 \mathrm{GHz}$ are depicted in Figure 10(c). The mix coupling was achieved by coupling between resonators 1 and 2, also, resonators 3 and 4, which are both in phase. Additionally, the mix coupling effects occurred by coupling between the resonators 1 and 3, as well as 2 and 4 are in phase with the magnetic coupling resulted by coupling between resonators 2 and 3. Also, the level of insertion loss is lower.

Figure 10(d) illustrates the coupling effects of our proposed filter with overlap resonators at the resonant frequency of $5.2 \mathrm{GHz}$. It was found that the mix, electric, and magnetic coupling effects are similar to those shown in Figure 10(c). However, the electric coupling, as shown in the electric fields, is that the results of the coupling between the resonators 1 and 3, as well as 2 and 4, and electric coupling, as in the coupling between the resonators 2 and 3 are in phase, resulting to increasing the coupling results of band-pass filter. Also, the level of insertion loss is improved to lower than $3 \mathrm{~dB}$ at the first harmonic resonant frequency of $5.2 \mathrm{GHz}$.

The appropriate parameters $D_{14}=0.23 \mathrm{~mm}, D_{23}=$ $0.74 \mathrm{~mm}, D_{12}=D_{34}=0.435 \mathrm{~mm}$, and $h=1.5 \mathrm{~mm}$ are chosen to design the proposed filter with overlap resonators, fabricated on a GML 1000 dielectric substrate with a thickness of $0.762 \mathrm{~mm}$, relative permittivity of $3.2\left(\varepsilon_{r}\right)$, and loss tangent of 0.004 . The size of the proposed filter with overlap resonator is approximately $2 \times 3 \mathrm{~cm}^{2}$, as depicted in the prototype of the proposed filter with overlap resonators (Figure 11). The fabricated filter was measured on an Agilent 8791ES network analyzer, with the simulated and measured results illustrated in Figure 12. It can be clearly seen that the simulated and measured results are in good agreement, with both the passband insertion losses of the fundamental and first harmonic resonant frequency at $2.4 \mathrm{GHz}$ and $5.2 \mathrm{GHz}$, which are approximately $1.5 \mathrm{~dB}$ and $2.2 \mathrm{~dB}$, respectively. As clearly evident from the results, the pass-band insertion loss of the first harmonic resonant frequency at $5.2 \mathrm{GHz}$ was improved because the in-phase mix coupling between overlap resonators was found by our investigation.

\section{Conclusions}

A dual-band band-pass filter using step-impedance and capacitively loaded hairpin resonators for Wireless LAN 
systems has been proposed and investigated. The proposed filter was compact in size, resulting from using the modified cross-coupling technique. Also, the first harmonic resonant frequency could be shifted by using the step-impedance and capacitively loaded resonator technique. Moreover, the technique of overlap resonator was used to improve the level of insertion losses, with approximately $2.2 \mathrm{~dB}$, at the first harmonic resonant frequency of $5.2 \mathrm{GHz}$. Therefore, the proposed filter can operate at the resonant frequencies of 2.4 GHz and 5.2 GHz for supporting the WLAN system.

\section{References}

[1] M. Makimoto and S. Yamashita, "Bandpass filter using parallel coupled stripline stepped impedance resonator," IEEE Transactions on Microwave Theory and Techniques, vol. 28, no. 12, pp. 1413-1417, 1980.

[2] A. A. A. Apriyana and Z. Y. Ping, "A dual-band BPF for concourrent dual-band wireless transceiver," in Proceedings of the Electronics Packaging Technology Conference, pp. 143-145, 2003.

[3] S.-F. Chang, Y.-H. Jeng, and J.-L. Chen, "Dual-band stepimpedance bandpass filter for multimode wireless LANs," IEEE Microwave and Wireless Components Letters, vol. 40, no. 1, pp. 38-39, 2004.

[4] J. T. Kuo, T. H. Yeh, and C. C. Yeh, "Design of microstrip bandpass filters with a dual-passband response," IEEE Transactions on Microwave Theory and Techniques, vol. 53, no. 4, pp. 13311336, 2005.

[5] Q. X. Chu and W. Ma, "A new dual-band step-impedance bandpass filter," in Proceedings of the IEEE Antennas and Propagation Society International Symposium and USNC/URSI Meeting, pp. 610-613, July 2005.

[6] H. Num, H. Lee, and Y. Lim, "A design and fabrication of bandpass filter using miniaturized microstrip square SIR," in Proceedings of the IEEE Region 10th International Conference on Electrical and Electronic Technology, pp. 395-398, August 2001.

[7] G. Dacheng, "Compact step impedance stripline bandpass filter," in Proceedings of the International Conference on Circuits and Systems, pp. 960-963, June 1991.

[8] E. G. Cristal and S. Frankel, "Hairpin-line and hybrid hairpinline/halfwaveparallel coupled-line filters," IEEE Transactions on Microwave Theory and Techniques, vol. 20, no. 11, pp. 719728, 1972.

[9] C. Quendo, J. P. Coupez, C. Person, E. Rius, M. Le Roy, and S. Toutain, "Band-pass filters with self-filtering resonators: a solution to control spurious resonances," in Proceedings of the IEEE MTT-S International Microwave Symposium Digest, pp. 1135-1138, June 1999.

[10] J. S. Hong, "Theory and experiment of novel microstrip slow-wave open-loop resonator filters," IEEE Transactions on Microwave Theory and Techniques, vol. 45, no. 12, pp. 23582365, 1997.

[11] J. S. Hong and M. J. Lancaster, "End-coupled microstrip slow-wave resonator filter," IEEE Microwave and Wireless Components Letters, vol. 32, no. 16, pp. 1494-1496, 1996.

[12] J. Gu, F. Zhang, C. Wang, Z. Zhang, M. Qi, and X. Sun, "Miniaturization and harmonic suppression open-loop resonator bandpass filter with capacitive terminations," in Proceedings of the IEEE MTT-S International Microwave Symposium Digest, pp. 373-376, June 2006.
[13] X. Zhang, Y. Wen, and K. Zhou, "A capacitive loaded quasi-elliptic function microstrip filter on GSM-R band," in Proceedings of the 3rd IEEE International Symposium on Microwave, Antenna, Propagation and EMC Technologies for Wireless Communications (MAPE '09), pp. 535-537, October 2009.

[14] G. L. Matthaei, L. Yong, and E. M. T. Jones, Microwave Filter, Impedance-Matching and Coupling Structures, McGraw-Hill, New York, NY, USA, 1964.

[15] J. S. Hong and M. J. Lancaster, "Cross-coupled microstrip hairpin-resonator filters," IEEE Transactions on Microwave Theory and Techniques, vol. 46, no. 1, pp. 118-122, 1998.

[16] J. S. Hong and M. J. Lancaster, "Couplings of microstrip square open-loop resonators for cross-coupled planar microwave filters," IEEE Transactions on Microwave Theory and Techniques, vol. 44, no. 11, pp. 2099-2109, 1996.

[17] S. Amari, "Synthesis of cross-coupled resonator filters using an analytical gradient-based optimization technique," IEEE Transactions on Microwave Theory and Techniques, vol. 48, no. 9, pp. 1559-1564, 2000.

[18] J. S. Wong, "Microstrip tapped-line filter design," IEEE Transactions on Microwave Theory and Techniques, vol. 27, no. 1, pp. 44-50, 1979.

[19] D. S. G. Chambers and J. D. Rhodes, "A low pass prototype allowing the placing of integrated poles at real frequencies," IEEE Transactions on Microwave Theory and Techniques, vol. 31, no. 1, pp. 40-45, 1983.

[20] IE3D Users Manual Release 8, Zeland Software, Inc., Fremont, Calif, USA, 2001. 

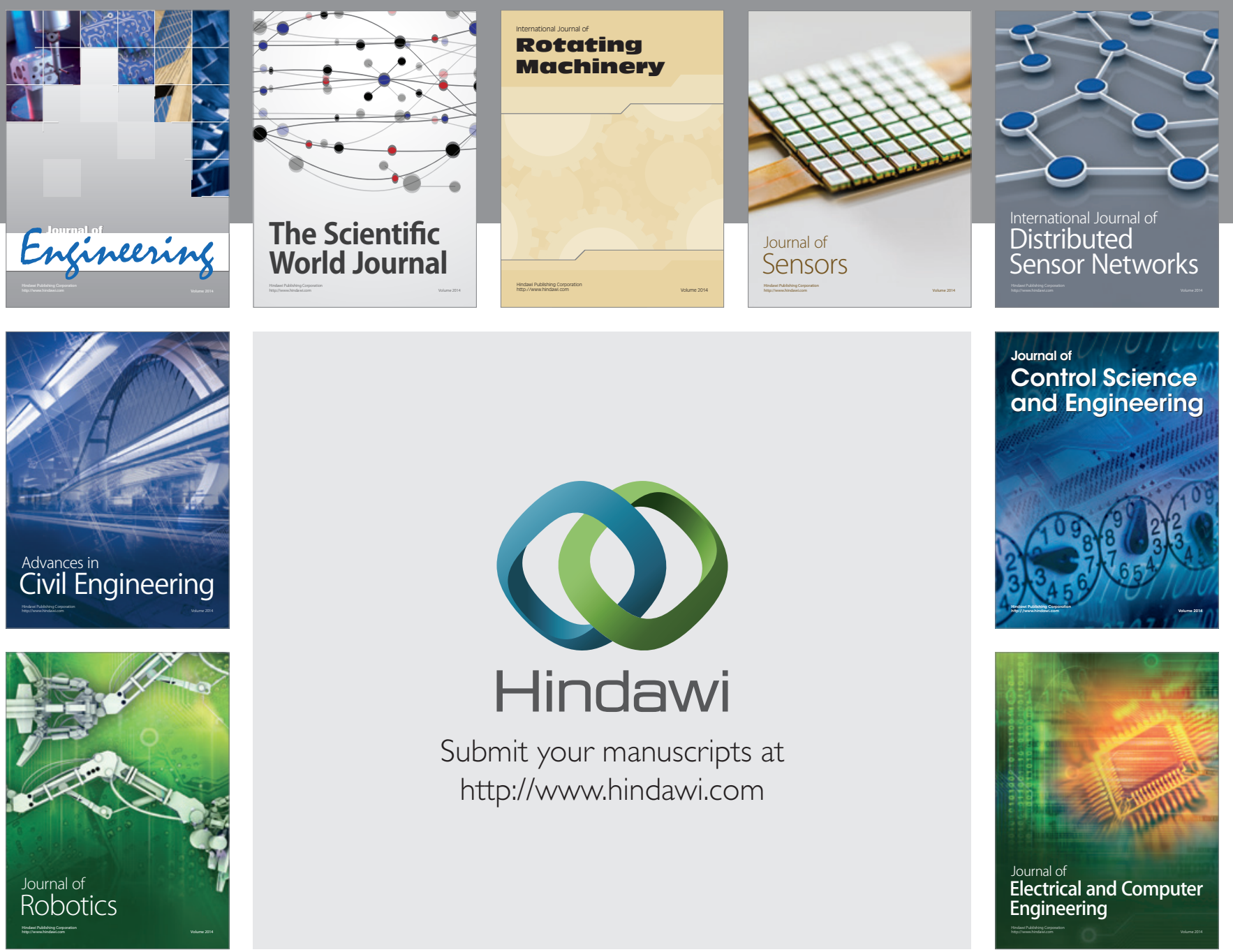

Submit your manuscripts at

http://www.hindawi.com
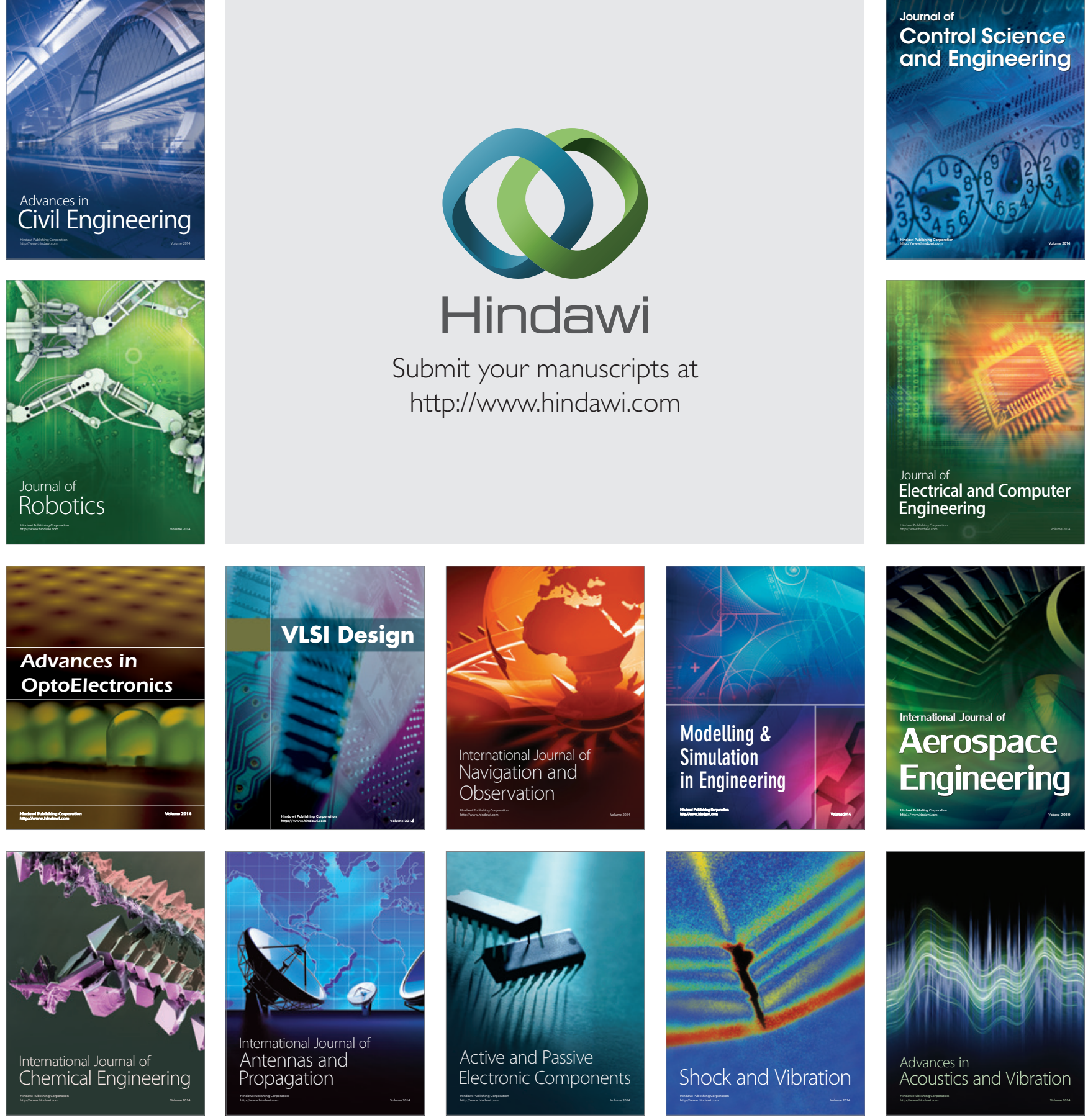